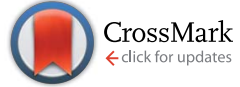

Cite this: J. Mater. Chem. A, 2014, 2 , 19495

Received 7th August 2014

Accepted 2nd October 2014

DOI: $10.1039 / \mathrm{c} 4 \mathrm{ta} 04076 \mathrm{~g}$

www.rsc.org/MaterialsA

\section{Nitrogen-doped and crumpled graphene sheets with improved supercapacitance $\dagger$}

\author{
Yuqin Zou, ${ }^{a}$ Ian A. Kinloch ${ }^{b}$ and Robert A. W. Dryfe ${ }^{* a}$ \\ Nitrogen-doped thermally expanded graphene oxide (NtGO) was prepared by a facile thermal expansion \\ and hydrothermal doping process. The thermal expansion process plays a vital role in improving the \\ electrochemical performance of $\mathrm{N}$-doped graphene by preventing its aggregation and improving its \\ conductivity. The specific capacitance of $\mathrm{NtGO}$ is $270 \mathrm{~F} \mathrm{~g}^{-1}$ at a discharge current density of $1 \mathrm{~A} \mathrm{~g}^{-1}$ and \\ the capacitance retention is $97 \%$ after 2000 cycles at this density. The strategy developed here provides \\ an efficient and facile way to prepare nitrogen-doped graphene.
}

Supercapacitors (also known as electrochemical capacitors (ECs) or ultracapacitors), represent a unique class of electrical energy storage devices, which has attracted increasing attention because of their high power density, fast charge-discharge rate and long cycle life. ${ }^{1}$ ECs can be classified into electrical doublelayer capacitors (EDLC) and pseudo-capacitors (PC). ${ }^{2}$ Due to their high power densities and long-term cycling stability, EDLCs have been extensively explored and become a promising device in high power electronic devices, electric vehicles (EVs) and hybrid electric vehicles (HEVs). ${ }^{3}$

Various carbon nanostructures have been widely investigated as electrode materials to improve the performance of supercapacitors. ${ }^{4-6}$ Graphene has been extensively used as an electrode material in supercapacitors, and researchers have recently developed many modification techniques to further increase its supercapacitive performance. ${ }^{7}$ Chemical functionalisation of graphene oxide, GO, is an effective method for manipulating physical and chemical properties of graphene, since enriched reactive oxygen functional groups in GO can provide ample covalent bonding sites for the chemical functionalisation. ${ }^{8}$ Moreover, it has been reported that nitrogen doping (or other heteroatom doping) could enhance the capacitance of graphene due to the modified electronic properties and the induced pseudo-capacitance in addition to the electrical double layer capacitance. .,5, $, \mathbf{6},-11,13,14$ Methods recently reported to synthesise nitrogen-doped graphene (NG), include annealing of graphene oxide with ammonia gas at high temperature, ${ }^{9}$ chemical vapor deposition, ${ }^{10}$ electrochemical exfoliation ${ }^{11,12}$ and plasma treatment. ${ }^{13}$ Compared with these

${ }^{a}$ School of Chemistry, University of Manchester, Oxford Road, Manchester M13 9PL, UK. E-mail: robert.dryfe@manchester.ac.uk

${ }^{b}$ School of Materials, University of Manchester, Oxford Road, Manchester M13 9PL, UK $\dagger$ Electronic supplementary information (ESI) available: Experimental section, the equivalent circuit model, cyclic voltammetry curves, galvanostatic charge-discharge curves for the samples, and capacitance retention. See DOI: 10.1039/c4ta04076g methods, the hydrothermal method has merits of higher nitrogen concentration, mild conditions and ready scale-up of the synthesis..$^{14}$ Kim and co-workers have prepared N-doped graphene by using a hydrothermal reaction with an amine reducing agent, resulting in a nitrogen content of $8.45 \%$. Freeze-drying was used to avoid aggregation, which can suppress the negative effect of the capillary forces during the drying process. However a long preparation time is required, usually over 48 hours. The resultant specific capacitance was $148 \mathrm{~F} \mathrm{~g}^{-1}$ at $1 \mathrm{~A} \mathrm{~g}^{-1}$. The aggregation of reduced graphene oxide is hard to avoid and dramatically decreases the surface area, leading to a poor capacitance. ${ }^{3}$ Designing a scalable process to prepare nitrogen-doped graphene without aggregation is therefore of paramount importance.

In this work, we used a facile thermal expansion method to pre-treat graphene oxide followed by nitrogen doping into graphene oxide with a hydrothermal process with hexamethylenetetramine (HMT) (the product was denoted as NtGO). The thermal expansion process makes the resultant graphene crumpled, which can prevent graphene oxide from aggregating during the subsequent drying process after hydrothermal doping. HMT acts as both reducing agent and $\mathrm{N}$ source for doping. Since the thermal expansion and the hydrothermal doping were both carried out at relatively low temperature, the method developed herein represents one that is suitable for large-scale industrial use. The capacitance of the resultant $\mathrm{NtGO}$ is $270 \mathrm{~F} \mathrm{~g}^{-1}$ at $1 \mathrm{~A} \mathrm{~g}^{-1}$, which is much higher than the conventional nitrogen doped graphene, attributed to the increased surface area by the thermal expansion and $\mathrm{N}$ doping.

The preparation of NtGO involves the pretreatment with a thermal expansion process (the intermediate product here is denoted as tGO) and hydrothermal nitrogen doping, as illustrated in Scheme 1. For comparison, we also prepared nitrogen doped graphene without the thermal expansion step to determine the effect of the thermal expansion on the supercapacitive performance of $\mathrm{N}$-doped graphene nanosheets. The volume 

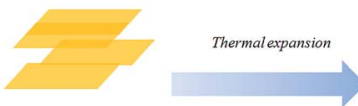

Graphene Oxide (GO)

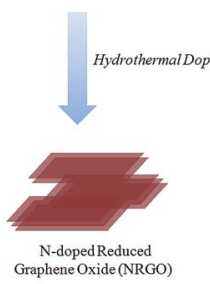

Scheme 1 Fabrication of $\mathrm{tGO}, \mathrm{NRGO}$ and $\mathrm{NtGO}$

change of a given weight of GO powder before and after the thermal expansion treatment is shown in Fig. 1a and b. It can be seen that after the thermal expansion at $800{ }^{\circ} \mathrm{C}$, the volume of graphene oxide powder was significantly expanded. Not only has the graphene powder in the glass bottle expanded, it is now a black and 'fluffy' tGO powder. This phenomenon implies that thermal expansion treatment could extend the volume and thus may expose a higher surface area of graphene. In order to observe the morphology of graphene samples in a direct way, scanning electron microscopy was performed on NRGO, tGO, as well as NtGO, as shown in Fig. 1c-e. GO can disperse quite well in water, giving a high concentration of monolayer GO
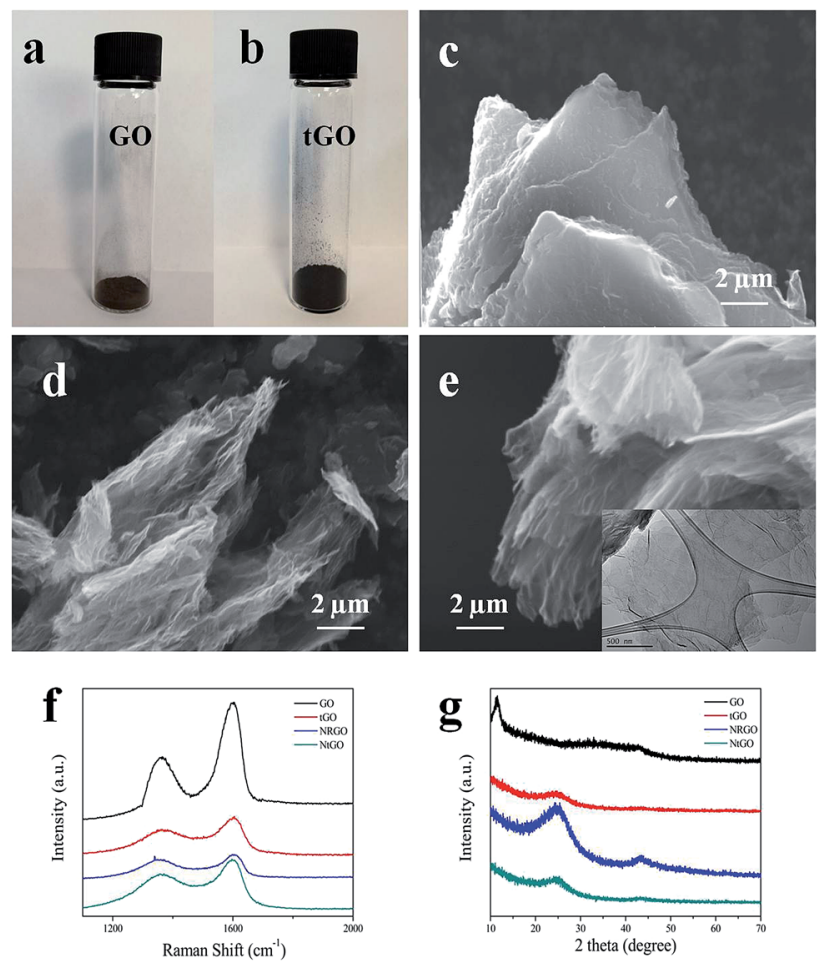

Fig. 1 Photographs of GO (a) before and (b) after thermal expansion. SEM images of (c) NRGO, (d) tGO and (e) NtGO. (f) Raman spectra of $\mathrm{GO}, \mathrm{tGO}, \mathrm{NRGO}$ and NtGO. (g) XRD patterns of GO, tGO, NRGO and NtGO. solution..$^{15}$ However, during the drying process after hydrothermal treatment, the well-dispersed GO readily aggregated to thick platelets, ${ }^{14}$ as shown in Fig. 1c. The thickness of the platelets is a few microns. This problem could be easily solved through a simple thermal expansion (for details, see the experimental section in the ESI $\dagger$ ). It is readily observed in Fig. 1d that the thin layer structure of $\mathrm{tGO}$ was preserved. Even after the subsequent hydrothermal process for nitrogen doping, the sheets keep the original thin layer structures (Fig. 1e, the inset is the TEM image). It has been well-recognized that there are many oxygen-containing groups on the surface of GO. ${ }^{16}$ Gases such as $\mathrm{CO}_{2}$ and $\mathrm{CO}$ were generated during thermal treatment at high temperature, leaving the material with spacing between two individual sheets. ${ }^{3}$ It should be pointed out that it is difficult to completely remove the oxygen-containing groups from the surface of GO by annealing it in $\mathrm{N}_{2} \cdot{ }^{16}$ The partially remaining oxygen species are very important for the subsequent $\mathrm{N}$-doping during the hydrothermal process. ${ }^{3}$ To demonstrate the expected role of the thermal expansion step, the surface area of NtGO and NRGO was measured by the Brunauer-Emmett-Teller (BET) analysis (nitrogen adsorption/ desorption). It is found that the surface area of $\mathrm{NtGO}$ is much higher than that of NRGO $\left(237 \mathrm{~m}^{2} \mathrm{~g}^{-1}\right.$ vs. $\left.141 \mathrm{~m}^{2} \mathrm{~g}^{-1}\right)$, indicating that thermal expansion could significantly increases the exposed surface area and suppresses the aggregation during the drying process. The increased surface area would potentially increase the supercapacitance of $\mathrm{N}$-doped graphene, as demonstrated below.

Raman spectroscopy is an efficient tool to characterize the structure and quality of carbon-based materials. The typical features in the Raman spectra of carbon materials are the D band at $1350 \mathrm{~cm}^{-1}$ and the $\mathrm{G}$ band at $1590 \mathrm{~cm}^{-1}$. The $\mathrm{D}$ band is associated with disordered samples or graphene edges, while the $\mathrm{G}$ band is the result of the first-order scattering of the $\mathrm{E}_{2 \mathrm{~g}}$ mode of $\mathrm{sp}^{2}$ carbon domains. ${ }^{16}$ To investigate the effect of the thermal expansion and the hydrothermal nitrogen doping, the Raman spectra of GO, tGO, NRGO and NtGO were collected, as shown in Fig. 1f. The intensity ratio of D band and $\mathrm{G}$ band is usually used as an indicator to investigate the defect levels of carbon. The values of $I_{\mathrm{D}}: I_{\mathrm{G}}$ of GO, tGO, NRGO and NtGO are $0.98,0.70,0.93$ and 0.75 , respectively. Obviously, $\mathrm{NtGO}$ shows a much lower $I_{\mathrm{D}}: I_{\mathrm{G}}$ ratio than NRGO, indicating fewer defects on the surface of graphene by the thermal expansion, which probably originates from the recovery of defects during the thermal expansion process or the removal of the functional groups of GO. By comparing the Raman spectra of tGO and $\mathrm{NtGO}$, it could be found that, after N-doping, the $I_{\mathrm{D}}: I_{\mathrm{G}}$ became larger because of the generation of more defects on the surface of graphene by N-doping. A slight shift of the G band of NtGO sample to higher wave numbers compared with that of tGO can be ascribed to the formation of chemical bonds between $\mathrm{C}$ and $\mathrm{N}$, which has been reported previously. ${ }^{13}$ However, the $I_{\mathrm{D}}: I_{\mathrm{G}}$ ratio value of NRGO is smaller than that of GO indicating that the degree of disorder of GO is stronger than that of NRGO. Fig. $1 \mathrm{~g}$ shows the XRD patterns of GO, tGO, NRGO and NtGO. The appearance of the diffraction peak at $11.8^{\circ}(002)$ is typical for GO samples corresponding to an interlayer spacing of 
$0.75 \mathrm{~nm} .{ }^{8}$ After thermal expansion, this peak shifted to about 24 degrees after reduction or doping for tGO, NRGO and NtGO. ${ }^{14}$ The corresponding interlayer spacing changes from $0.75 \mathrm{~nm}$ for GO to $0.36 \mathrm{~nm}$ for tGO, NRGO and NtGO, indicating the few layer stacked graphene nanosheet of their frameworks and the recovery of a more graphitic structure. ${ }^{\mathbf{1 6}}$ During the hydrothermal process, HMT plays a role both as reducing agent and $\mathrm{N}$ source. HMT can reduce GO by removing oxygen-containing functional groups and releases ammonia which can simultaneously introduce nitrogen atoms into the graphene skeleton by substituting for carbon atoms. ${ }^{12}$ XPS characterization was performed in order to analyze the elemental compositions and nitrogen bonding configuration in NtGO. All the peaks were corrected to C1s (284.8 eV). For the XPS survey spectra both for tGO and NtGO shown in Fig. 2a, the peaks at around $284.8 \mathrm{eV}$ and $533.5 \mathrm{eV}$ can be assigned to the binding energies of $\mathrm{C} 1 \mathrm{~s}$ and O1s respectively. ${ }^{9}$ As shown in Fig. 2 a, a N1s peak at $399.8 \mathrm{eV}$ is observed in the XPS spectra of NtGO. The calculated atomic percentage of $\mathrm{N}$ in $\mathrm{NtGO}$ was $5.8 \%$ (the atomic content of GO, tGO, NRGO and NtGO is listed in Table S1 $\dagger$ ). To investigate the chemical state, the C1s and N1s XPS peak of NtGO sample were fitted, as given in Fig. $2 \mathrm{~b}$ and c. The C1s peak of NtGO can be fitted to four components, corresponding to the $\mathrm{C}=\mathrm{C} / \mathrm{C}-\mathrm{C}$, $\mathrm{C}-\mathrm{N}, \mathrm{C}=\mathrm{O}$, and $\mathrm{O}-\mathrm{C}=\mathrm{O}$ species located at 284.8, 285.9, 287.7 and $291.0 \mathrm{eV}$ respectively. ${ }^{15}$ The fractions of $\mathrm{C}=\mathrm{C} / \mathrm{C}-\mathrm{C}, \mathrm{C}-\mathrm{N}$, $\mathrm{C}=\mathrm{O}, \mathrm{O}-\mathrm{C}=\mathrm{O}$ is $0.65,0.19,0.12,0.04$ respectively. Similarly, the N1s XPS peaks could be fitted into three components (Fig. 2c). The peak at $\sim 398.4 \mathrm{eV}$ corresponds to pyridinic $\mathrm{N}$, and the peak at $399.8 \mathrm{eV}$ can be indexed to pyrrolic $\mathrm{N}$. The peak at $\sim 401 \mathrm{eV}$ is assigned to quaternary $\mathrm{N},{ }^{5}$ indicating that nitrogen replaced the carbon atom in the graphene sheets and was incorporated into the carbon network.
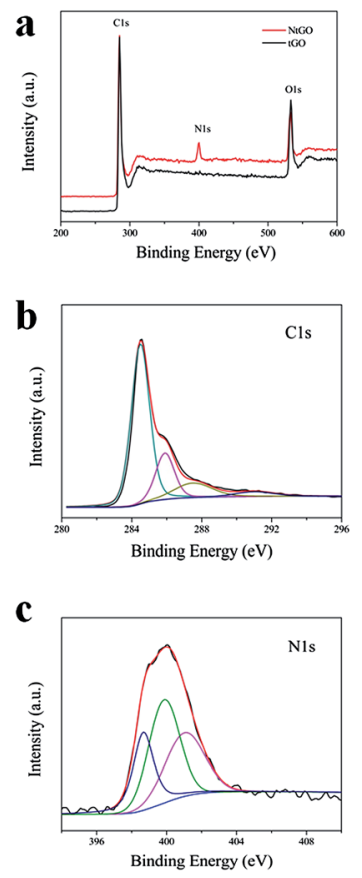

Fig. 2 (a) XPS spectra of $\mathrm{tGO}$ and NtGO samples. (b) high-resolution C1s XPS spectra of NtGO. (c) high-resolution N1s XPS spectra of NtGO.
The above characterization results demonstrate the unique crumpled structure of NtGO and high nitrogen doping content. The nitrogen species of HMT have reacted with carbon atoms on the defects and vacancies, or replaced the carbon atoms in the lattice of graphene. Compared with previously reported routes to nitrogen-doped graphene, ${ }^{\mathbf{1 2 - 1 4}}$ the thermal expansion pre-treatment can give a crumpled structure to graphene, which can suppress the aggregation of graphene sheets during the subsequent hydrothermal doping process and the consecutive hydrothermal process enabled the nitrogen doping reaction to proceed on both sides of GO, leading to uniform nitrogen doping in graphene. This differs from the NG prepared using ammonia gas as the nitrogen source, in which the reaction mostly occurred on the exposed surface of carbon nanostructures, thereby resulting in inhomogeneous ${ }^{3}$ and low nitrogen doping, of the order of $2-3$ at $\% .^{11}$

In order to investigate the effect of the thermal expansion on the supercapacitance of nitrogen-doped graphene nanosheets, cyclic voltammetry (CV), galvanostatic charge-discharge, and electrochemical impedance spectroscopy (EIS) tests were carried out using a three-electrode cell in $1 \mathrm{M} \mathrm{H}_{2} \mathrm{SO}_{4}$ electrolyte with $\mathrm{Pt}$ mesh and $\mathrm{Ag} / \mathrm{AgCl}$ (saturated $\mathrm{KCl}$ solution) as the counter electrode and reference electrode respectively. Fig. 3a shows the CV curves of different electrodes at the scan rate of 20 $\mathrm{mV} \mathrm{s}^{-1}$. There is no distinctive peak in the CV curve of tGO while a pair of redox peaks in the CV curves of NRGO and NtGO was observed, which are caused by the nitrogen doping induced pseudo-capacitance. ${ }^{8}$ The rectangular $\mathrm{CV}$ curves indicate the ideal double layer capacitor nature with a charge-discharge process. Additionally, the cell with NtGO electrode (red line) exhibits much larger current than the electrode of tGO (black) and NRGO (blue), indicating increased capacitance due to thermal expansion. Cyclic voltammetry of tGO, NRGO and
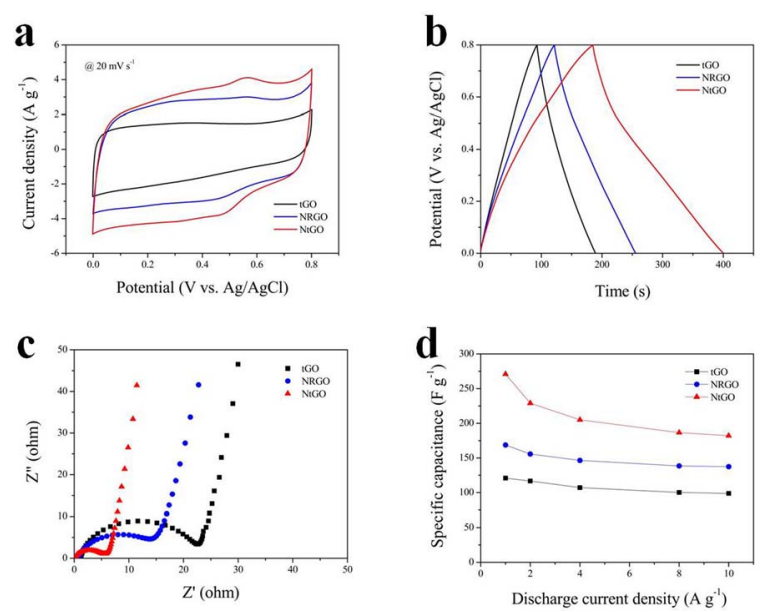

Fig. 3 Electrochemical performance of tGO, NRGO and NtGO using a three-electrode cell in $1 \mathrm{M} \mathrm{H}_{2} \mathrm{SO}_{4}$. (a) $\mathrm{CV}$ s of as-prepared samples at the scan rate of $20 \mathrm{mV} \mathrm{s}^{-1}$. (b) Galvanostatic charge-discharge curves of as-prepared samples at discharge current density of $1 \mathrm{~A} \mathrm{~g}^{-1}$. (c) Nyquist plots of as-prepared samples $(0.01 \mathrm{~Hz}$ to $100 \mathrm{KHz}$, an AC amplitude, $5 \mathrm{mV}$ ). (d) Specific capacitance of as-prepared samples at different discharge current densities. 
NtGO at various scan rates are shown on Fig. S2, S4 and S6. $\dagger$ The improved capacitance of NtGO can also be observed from the galvanostatic charge-discharge curves at a discharge current density of $1 \mathrm{~A} \mathrm{~g}^{-1}$ as shown in Fig. 3b. The specific capacitance of NtGO is $270 \mathrm{~F} \mathrm{~g}^{-1}$ at the current density of $1 \mathrm{~A} \mathrm{~g}^{-1}$, while those of tGO and NRGO are 121 and $169 \mathrm{~F} \mathrm{~g}^{-1}$ respectively. The energy density $(E)$ of NtGO at current density of $1 \mathrm{~A} \mathrm{~g}^{-1}$ is $24 \mathrm{Wh}$ $\mathrm{kg}^{-1}$. The galvanostatic charge-discharge curves of as-prepared samples at various current densities are shown on Fig. S3, S5 and S7. $\dagger$ Electrochemical impedance spectroscopy (EIS) was utilized to further investigate the role of nitrogen-doping in enhancing the capacitive performance of the samples. Nyquist plots in Fig. 3c consist of a semicircle in the high-frequency region and a line in the low-frequency region corresponding to a diffusion process. The semicircle of NtGO in the high frequency region is smaller than that of other two samples, indicating the equivalent series resistance (ESR) of NtGO is lower than other two samples. The equivalent circuit model fitted with the Autolab (Metrohm UK, Runcorn) NOVA software is shown on Fig. $\mathrm{S} 1 \uparrow$ and the parameters fitted from EIS spectra are listed in Table $\mathrm{S} 2 . \dagger R_{\mathrm{S}}, C_{\mathrm{dl}}, R_{\mathrm{F}}, C_{\mathrm{F}}$ and $W$ represent the solution resistance, double-layer capacitance, Faradaic resistance, pseudocapacitance and Warburg impedance, respectively. The ESR of NtGO is $0.11 \mathrm{ohm}$, with which we can obtain a power density $(P)$ of $1.5 \mathrm{~kW} \mathrm{~kg}^{-1}$. Fig. 3d shows the dependence of the specific capacitance of as-prepared samples on the discharge current densities. The specific capacitance of NtGO is $230 \mathrm{~F} \mathrm{~g}^{-1}$ at the current density of $2 \mathrm{~A} \mathrm{~g}^{-1}$. Due to the synergistic effects of high surface area and N-doping, the specific capacitance of NtGO is still as high as $182 \mathrm{~F} \mathrm{~g}^{-1}$ even at the current density of $10 \mathrm{~A} \mathrm{~g}^{-1}$, indicating that the NtGO electrode shows a good rate capability. The electrochemical stability of the NtGO electrode for supercapacitor application was also evaluated using the galvanostatic charge-discharge technique at a current density of $1 \mathrm{~A} \mathrm{~g}^{-1}$ (Fig. S8 $\dagger$ ). The capacitance retention of NtGO is $97 \%$ after 2000 cycles. The results again highlight that NtGO has excellent electrochemical stability and a high degree of reversibility.

The above results clearly reveal that NtGO exhibits significantly improved electrochemical performance compared with tGO and NRGO. It should be noted that the NtGO has a high nitrogen doping content (the ratio of $\mathrm{C}$ to $\mathrm{N}$ is 15.97) with apparent crumpled structures which can help it achieve the important aspects of high capacity, excellent rate capability and cycle performance. There are four possible reasons for the improved supercapacitance: firstly, pyridinic $\mathrm{N}$ and pyrrolic $\mathrm{N}$ are assumed to be the main configurations contributing to the Faradic reaction-based pseudo-capacitance. It is also reported that the presence of quaternary $\mathrm{N}$ can enhance the conductivity of the materials, which is beneficial for the supercapacitor performance. ${ }^{1}$ Additionally, introducing nitrogen into carbonaceous electrodes can change the electron distribution of the materials, which can further enhance the wettability between electrode materials and electrolyte, leading to a significant increase of active surface area accessible to the electrolyte. ${ }^{15}$ Thirdly, NtGO has a high atomic percentage of oxygen (10\%) indicating there were surface functional groups such as hydroxyl and carbonyl groups which can provide pseudo- capacitance as a complement to the capacitance. ${ }^{17}$ Finally, annealing at high temperature can increase the conductivity of carbon-based materials, giving a highly crumpled structure. It can provide a high accessible specific surface area for adsorbing ions and accelerating electron transfer or decreasing electric resistance loss. ${ }^{3}$ Moreover, we compared our results with reported data on nitrogen-doped carbons (ESI, Table S3†). We found the capacitance of the samples synthesised with our simple method to be higher than most reported values. To the best of our knowledge, this is the first reported preparation of nitrogen-doped graphene by combining a thermal expansion pre-treatment and hydrothermal doping method. The thermally expanded process can prevent sample aggregation and increase the conductivity of the sample, the consecutive hydrothermal process can give rise to a high $\mathrm{N}$ doping level.

\section{Conclusions}

In conclusion, a facile thermal expansion process was employed to prepare crumpled nitrogen doped graphene with the aim of achieving enhanced supercapacitive performance. Thermal expansion plays a vital roles in preventing doped graphene from aggregating and improving the capacitance of the nitrogendoped graphene. Our physical characterizations demonstrated that the thermal expansion pre-treatment could increase the surface area of the electrode materials significantly. The NtGO electrode showed an excellent supercapacitive performance attributed to a combination of thermal expansion and $\mathrm{N}$-doping. The method developed here is a facile way to prepare $\mathrm{N}$-doped graphene with high surface area and thus high specific capacitance, and is compatible with scale-up due to the simplicity and efficiency of the preparation.

\section{Acknowledgements}

We thank EPSRC (grant ref: EP/K016954/1) for support and the University of Manchester President's Doctoral Scholarship for a studentship for Y.Z.

\section{Notes and references}

1 L. Hao, X. Li and L. Zhi, Adv. Mater., 2013, 25, 3899.

2 B. E. Conway, Electrochemical Supercapacitor, Kluwer Academic/Plenum Publisher, New York, 1999.

3 Z. Wen, X. Wang, S. Mao, Z. Bo, H. Kim, S. Cui, G. Lu, X. Feng and J. H. Chen, Adv. Mater., 2012, 24, 5610.

4 Y. Zhu, S. Murali, M. D. Stoller, K. J. Ganesh, W. Cai, P. J. Ferreira, A. Pirkle, R. M. Wallace, K. A. Cychosz, M. Thommes, D. Su, E. A. Stach and R. S. Ruoff, Science, 2011, 332, 1537.

5 P. Chen, J. Yang, S. Li, Z. Wang, T. Xiao, Y. Qian and S. H. Yu, Nano Energy, 2013, 2, 249.

6 Z. Lei, L. Lu and X. Zhao, Energy Environ. Sci., 2012, 5, 6391.

7 L. Dai, Acc. Chem. Res., 2013, 46, 31.

8 S. J. Park and R. S. Ruoff, Nat. Nanotechnol., 2009, 4, 217. 
9 L. L. Zhang, X. Zhao, H. X. Ji, M. D. Stoller, L. F. Lai, S. Murali, S. Mcdonnell, B. Cleveger, R. M. Wallacec and R. S. Ruoff, Energy Environ. Sci., 2012, 5, 9618.

10 Z. Jin, J. Yao, C. Kittrell and J. M. Tour, ACS Nano, 2011, 5, 4112.

11 J. Liu, C. K. Poh, D. Zhan, L. Lai, S. H. Lim, L. Wang, X. Liu, N. Gopal Sahoo, C. Li, Z. Shen and J. Lin, Nano Energy, 2013, $2,377$.

12 J. Liu, H. Yang, S. G. Zhen, C. K. Poh, A. Chaurasia, J. Luo, X. Wu, E. K. L. Yeow, N. G. Sahoo, J. Lin and Z. Shen, RSC Adv., 2013, 3, 11745.
13 H. M. Jeong, J. W. Lee, W. H. Shin, Y. J. Choi, H. J. Shin, J. K. Kang and J. W. Choi, Nano Lett., 2011, 11, 2472.

14 J. W. Lee, J. M. Ko and J. D. Kim, Electrochim. Acta, 2012, 85, 459.

15 H. L. Cao, X. F. Zhou, Z. H. Qin and Z. P. Liu, Carbon, 2013, 56, 218.

16 D. H. Deng, X. L. Pan, L. Yu, Y. Cui, Y. P. Jiang, J. Qi, W. X. Li, Q. Fu, X. C. Ma, Q. K. Xue, G. Q. Sun and X. H. Bao, Chem. Mater., 2011, 23, 1188.

17 F. Liu and D. F. Xue, Chem.-Eur. J., 2013, 19, 10716. 